\title{
Three-dimensional analysis of silver nano-particles doping effects on super resolution near-field structure
}

\author{
Yuan-Fong Chau ${ }^{\mathrm{a}, *}$, Din Ping Tsai ${ }^{\mathrm{b}}$ \\ ${ }^{a}$ Department of Electronic Engineering, Chin Yun University, Jung-Li 320, Taiwan, ROC \\ ${ }^{\mathrm{b}}$ Department of Physics and Center for Nanostorage Research, National Taiwan University, Taipei, Taiwan, ROC
}

Received 22 April 2006; received in revised form 23 July 2006; accepted 5 August 2006

\begin{abstract}
The super resolution near-field structure which incorporates a AgOx thin film was studied through the calculation using three-dimensional (3D) finite-difference time-domain method. The influences of the optical field distribution generate by some factors, e.g., the polarization direction, the wavelength of incident light and the size of silver nano-particles, which are sensitive to the surface plasmon resonance are discussed in detail. The goal of this study is to explain the physical mechanisms responsible for the super-resolution near-field structure phenomena in 3D model and give a better understanding of the optical properties between AgOx layer and incident light.
\end{abstract}

(C) 2006 Elsevier B.V. All rights reserved.

PACS: 42.79.Vb; 71.15.Rn; 72.15.Rn; 73.22.-f; 73.22.Lp; 78.67.Bf; 73.20.Mf

The achievable storage density of conventional optical data storage system is ultimately confined by diffraction limit. Currently, there is great interest in techniques that may overcome this limit. One of the promising techniques is using a storage medium which employs a super-resolution near-field structure (Super-RENS [1-7]). A SuperRENS structure is composed of an active layer that is sandwiched between two dielectric films, and this three layer stack is deposited directly on the phase change data layer of the disk. The dielectric layer is directly adjacent to the polycarbonate (PC) layer as the insulating layer; the other dielectric layer is the cover layer. If a storage medium incorporates a Super-RENS structure, the light spot that probes the data layer is smaller than the size of the focused spot that is incident on the cover layer of the Super-RENS structure. It is believed that this occurs because a small light-transmitting aperture is formed only in those regions of the Super-RENS active layer illumi-

\footnotetext{
* Corresponding author. Tel.: +886 34581196x51; fax: +886 34588924. E-mail address: yfc01@cyu.edu.tw (Y.-F. Chau).
}

nated by portions of the incident focused light spot that have sufficiently high intensity.

Currently, two materials are being used to form the Super-RENS active layer; these are antimony $(\mathrm{Sb})$ and silver-oxide (AgOx). The mechanism of $\mathrm{Sb}$ which forms the aperture is perhaps well known $[2,8]$. Sb changes its phase under heating [8] and thus a small region that exhibits a reduced index of refraction (i.e., an aperture) can be formed proximate to the central (high intensity) part of the incident focused beam. However, with AgOx the mechanism that forms such an aperture is not clearly discussed in three-dimensional (3D) model. The resolution provided by the two-dimensional (2D) model Super-RENS [1,9-11] is lower than that expected on the basis of experiments. Of the several possible reasons, the soft decay of the fields in 2D $\left(r^{-1 / 2}\right.$ instead of $\left.r^{-1}\right)$ might be the most fundamental reason. This shows the need for 3D calculations which, however, require much computation times. It has been suggested that small (on the order of $1 \mathrm{~nm}$ ) colloidal silver (Ag) particles are locally formed in the $\mathrm{AgOx}$ layer where it is sufficiently heated. Surface plasmons produced on the surface of these particles could then enhance the optical 
field strength in a small (relative to incident focused spot) region directly behind the Super-RENS structure (see, e.g., Ref. [1]).

A 3D finite-difference time-domain (FDTD) model has been used to compute the optical fields that occur at the phase change layer of a storage medium that incorporates a Super-RENS structure which has a AgOx in the heated active layer. The goal of this paper is to find the influences of various factors, such as the polarization direction, the wavelength of incident wave, and various size of silver nano-particles, which produce an optical field on the phase change layer that is significantly smaller in size than the incident focused spot and which has appreciable intensity. If such a spatial distribution of materials within the SuperRENS active layer that produces the desired results are able to find, it would enable us to model the Super-RENS writing and reading process and to better understand the Super-RENS phenomenon. In addition, by manipulating the localized surface plasmon resonance, local field enhancement, and near-field coupling of nano-particles, it is promising to be able to focus, to transport, and to interact with light in nano-scale region and are being explored for various applications in chemical and biological sensors [12], nano-photonic devices [13], plasmonics [14], etc.

Fig. 1 shows the Super-RENS storage medium structures. The layer structure is from bottom to top Polycarbonate $(n=1.5, h=150 \mathrm{~nm}$, where $h$ is the thickness of layer $) / \mathrm{ZnS}-\mathrm{SiO}_{2}(n=2.243, h=20 \mathrm{~nm}) / \mathrm{AgOx}(n=2.261$, $h=15 \mathrm{~nm}) / \mathrm{ZnS}-\mathrm{SiO}_{2}(h=20 \mathrm{~nm}) / \mathrm{Ge}_{2} \mathrm{Sb}_{2} \mathrm{Te}_{5}$ (GST, crystal phase, $n_{\mathrm{c}}=4.01+\mathrm{i} 3.61$, amorphous phase, $n_{\mathrm{a}}=4.45$ $+\mathrm{i} 1.65, h=15 \mathrm{~nm}) / \mathrm{ZnS}-\mathrm{SiO}_{2} \quad(h=20 \mathrm{~nm}) / \mathrm{air} \quad(n=1)$. The nano-size Ag particles are embedded in the $\mathrm{AgOx}$ layer either by the fabrication process or by the photo dissociation process with incident light. We assume that the $\mathrm{Ag}$ particles are of the same size and randomly distributed in the AgOx layer as shown in Fig. 1. The dispersive behavior of silver and GST is simulated by the Lorentz model [15], where the resonant frequency $\left(\omega_{0}\left(s^{-1}\right)\right)$ of $\mathrm{Ag}$, GST (normal), GST(mark) are $1.41477 \times 10^{15}, 3.57 \times 10^{15}$, $2.9739 \times 10^{15}$ and damping coefficient $\Gamma\left(s^{-1}\right)$ of $\mathrm{Ag}$, GST(normal), GST(mark) are $1.9378 \times 10^{13}, 1.548 \times 10^{15}$, $1.1844 \times 10^{15}$, respectively. We set the DC response of the polarization to the electric field $\chi_{0}=10$ and $\varepsilon_{\infty}=2.98 \varepsilon_{0}$ in this work. The $\mathrm{AgOx}$ layer is sandwiched between insulating $\mathrm{ZnS}-\mathrm{SiO}_{2}$ layers and a $\mathrm{Ge}_{2} \mathrm{Sb}_{2} \mathrm{Te}_{5}$ (GST) alloy phase change data layer is located directly under this tri-layer structure. The incident write/read light travels in the bottom (cover) $\mathrm{ZnS}-\mathrm{SiO}_{2}$ layer. A Gaussian source (located in the $\mathrm{ZnS}-\mathrm{SiO}_{2}$ cover layer and implemented as a $\mathrm{SF} /$ TF source) and the phase change data layer were introduced. The others parameters that were used are polarization: s-polarization and p-polarization, cell size $\Delta x=\Delta y=$ $2 \mathrm{~nm}$ and $\Delta z=0.5 \mathrm{~nm}$, the refractive index of $\operatorname{Ag} n=0.7+$ i3.65, the incident Gaussian beams FWHM was adjusted such that $\mathrm{FWHM}=0.51 \lambda / \mathrm{NA}[16]$ and the phase change data layer is on-mark situation. The on-mark situation meant that the incident light focuses on the recording mark. The effects of light polarization on the generated near-field images are studied under both parallel and perpendicular polarization conditions. When the model structure possesses a preferred orientation, the characteristics of the scattered light depend on the polarization direction (either parallel or perpendicular) relative to the structure orientation. In this work, we report that the incident

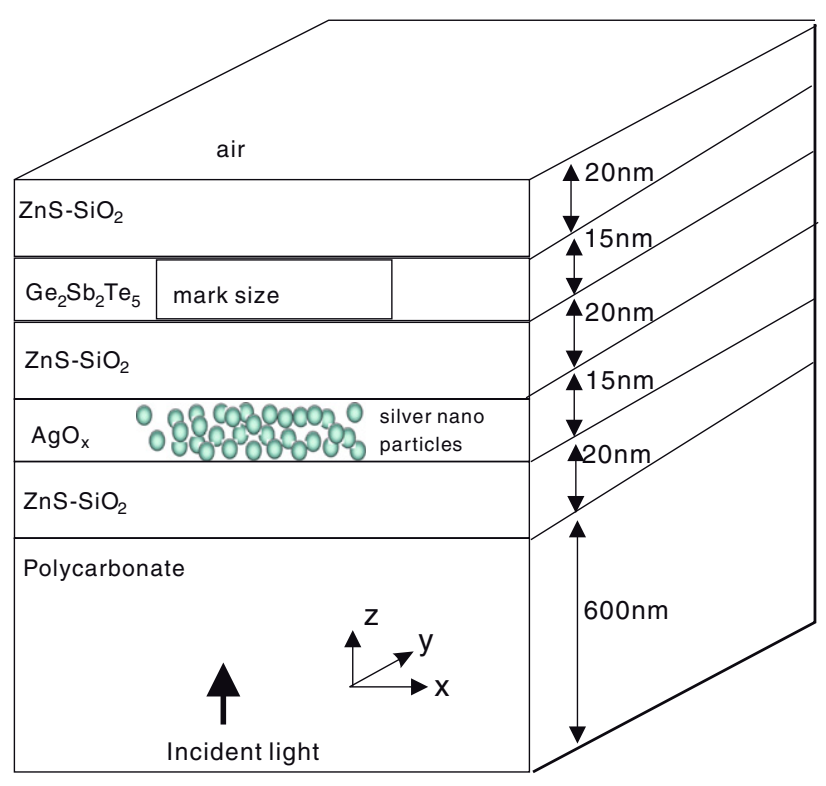

a

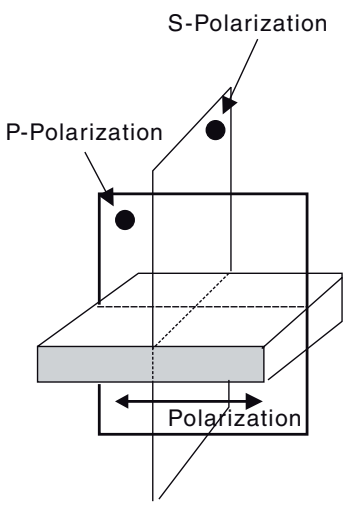

b

Fig. 1. (a) Schematic diagram of a modelled Super-RENS configuration. (b) Convention used when considering the effects of two orthogonal polarization states (s- and p-polarizations). The incident electric field is p-polarized when the electric field vector oscillates in a sectional plane that is perpendicular to the surface of the edge structure. On the other hand, s-polarization occurs when the field vector oscillates in the normal direction to a sectional plane. 
electric field is p-polarized when the electric field vector oscillates in a sectional plane that is perpendicular to the surface of the edge structure. On the other hand, s-polarization occurs when the field vector oscillates in the normal direction to a sectional plane.

Fig. $2 \mathrm{a}$ and $\mathrm{b}$ show the calculated results of the $3 \mathrm{D}$ near-field distribution (contour map of $E_{y}$ in $x-z$ plane at $y=0$ ) for s-polarization (Fig. 2a) and p-polarization (Fig. 2b) illuminations, respectively. The diameter of $\mathrm{Ag}$ nano-particles is $d=5 \mathrm{~nm}$ and the wavelength of incident wave is $\lambda=650 \mathrm{~nm}$. Although this model is a rotational symmetric system, the electric field distributions formed in the two orthogonal cross sections are different from each other due to the difference between the boundary conditions at the edge interface. In the sectional image plane shown in Fig. 2, highly localized enhancements were produced between adjacent Ag nano-particles in the near-field zone only for p-polarization (Fig. 2b). Where the white color presents the field intensity value larger than that the maximum color used in color scale in the range of $[1,-1]$. The field enhancement is due to the surface plasmon polarization. Each embedded Ag nano-particles
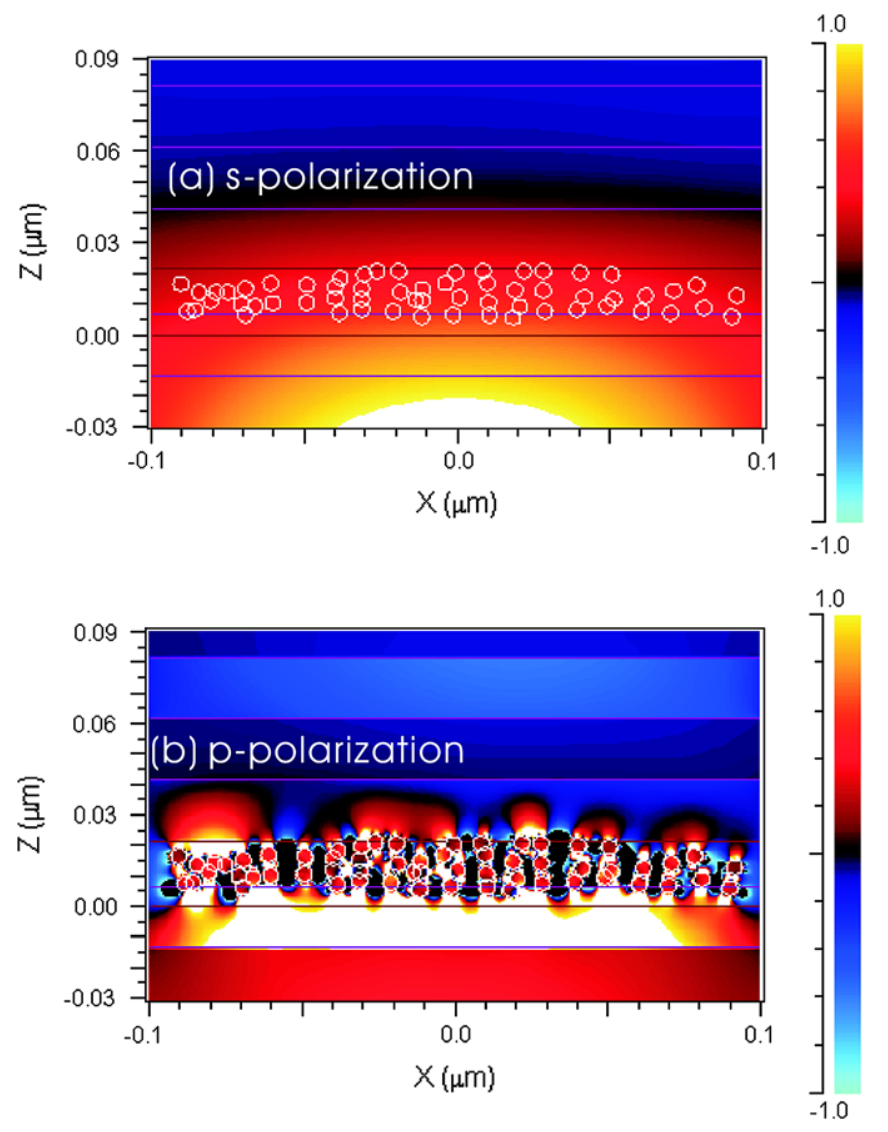

Fig. 2. Calculated results of the $3 \mathrm{D}$ near-field distribution (contour map of $E_{y}$ in $x-z$ plane at $y=0$ ) for (a) p-polarization and (b) s-polarization illuminations, respectively, where the diameter of $\mathrm{Ag}$ nano-particles is $5 \mathrm{~nm}$ and the wavelength of incident wave is $\lambda=650 \mathrm{~nm}$. The electric field results from an incident wave coming from the bottom side of the model shown in Fig. 1. behaves like a dipole and a very strong scattering center. The local fields from adjacent nano-particles are coupled together and produce highly enhanced evanescent fields. When free electrons of Ag nano-particles oscillate collectively in resonance with the incident light, the interaction may constitute a surface plasmon polaritons (SPPs). Since the boundary conditions of Maxwell's equations state that induced surface charge density is proportional to the discontinuity of the electric field component normal to the surface, only p-polarized light can excite SPPs in this case. These near-field optical signals of Ag nano-particles corresponded to the nonlinear near-field optical properties which were previously found in experiments [17].

Surface plasmon resonance is usually sensitive to the different wavelength of incident light. For simple Ag nanoparticles, strong light absorbing and scattering occurs at plasmon resonance frequency and high local fields were excited around the nano-particles [18]. However, in a system consisting of randomly distributed Ag nano-particles, the surface plasmon broaden and resonant condition is modified for particle-particle interaction. It is necessary to investigate the wavelength-dependent responses of the AgOx-type super-RENS disk to understand the roles of surface plasmon effect and particle-particle interaction, especially in 3D model. Fig. 3 shows the simulation results of field intensity of $\mathrm{Ag}$ nano-particles in the $\mathrm{AgOx}$ layer as a function of wavelength of incident light that corresponds to diameters of $\mathrm{Ag}$ nano-particles of $3 \mathrm{~nm}$ and the field intensity is measured at the top surface of $\mathrm{AgOx}$ layer. The wavelength of incident light is increased from $300 \mathrm{~nm}$ to $850 \mathrm{~nm}$ with increment of $5 \mathrm{~nm}$ for p-polarization. It is clearly seen that the field intensity of silver

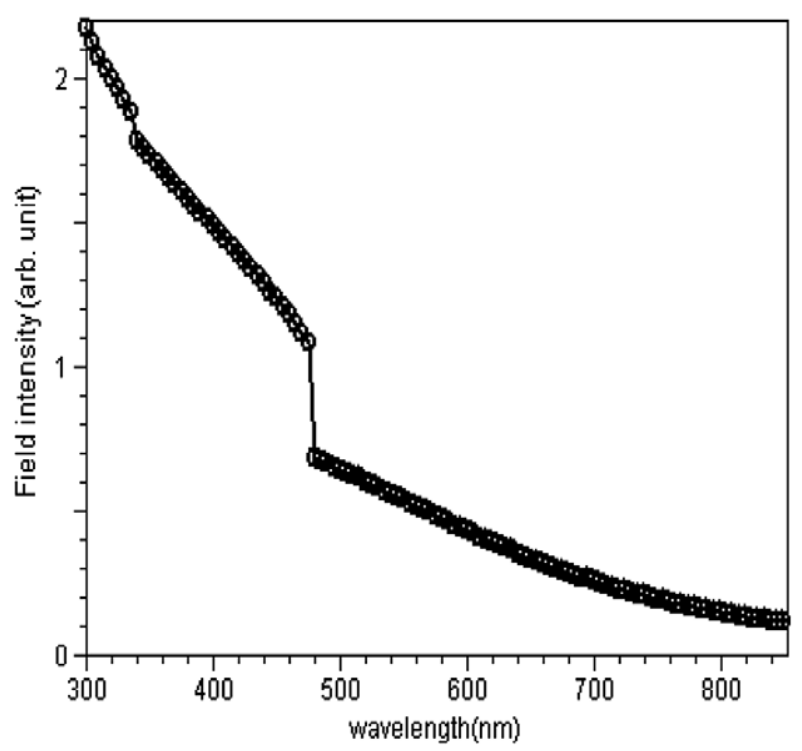

Fig. 3. The field intensity of silver nano-particles in the AgOx layer as a function of wavelength of incident light corresponds to diameters of silver nano-paticles of $3 \mathrm{~nm}$. The field intensity is measured at the top surface of AgOx layer. The wavelength of incident light is increased from $300 \mathrm{~nm}$ to $850 \mathrm{~nm}$ with increment of $5 \mathrm{~nm}$ for p-polarization. 
nano-particles is decreased as the wavelength of incident light increased and abruptly descends at wavelength of $335 \mathrm{~nm}$ and $475 \mathrm{~nm}$.

To verify the effect of nano-particle size, the field intensity of Ag nano-particles with increasing diameter was test. Fig. 4 shows the field intensity as a function of the diameters of silver nano-particles from $0 \mathrm{~nm}$ (no particle) to $12 \mathrm{~nm}$ with increment of $1 \mathrm{~nm}$ for (a) p-polarization and s-polarization, respectively. We chose the wavelength of incident light of $405 \mathrm{~nm}, 550 \mathrm{~nm}, 650 \mathrm{~nm}$ and $750 \mathrm{~nm}$, respectively as examples, and the surface plasmon effects of other wavelengths can be verified by the same procedures. The results clearly show that the size variation of silver nano-particles may yield different field intensity and give a better understanding of the optical properties of the $\mathrm{AgOx}$ type Super-RENS. For p-polarization in Fig. 4a, when nano-particle diameter was less than $1 \mathrm{~nm}$, i.e., the surface area of $\mathrm{Ag}$ nano-particles are too small that can excite less evanescent field from the surface, the effect of surface plasmon resonance became weaker since the evanescent light generated by smaller diameter of $\mathrm{Ag}$ nano-particles was transferred to propagating light. When the particle size was larger than $7 \mathrm{~nm}$, the Ag nano-particles form aggregative silver clusters which act as a silver bulk. The intensity decreases
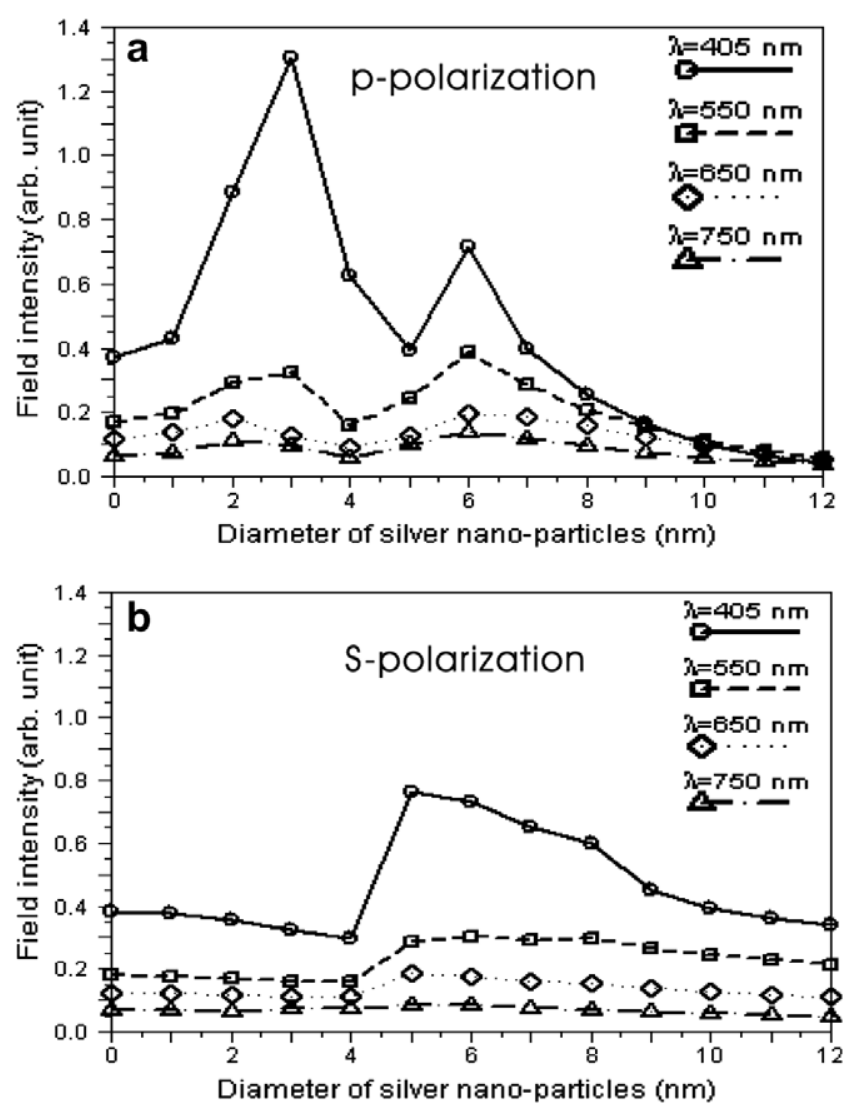

Fig. 4. The field intensity at different wavelengths as a function of the diameters of silver nano-particles from $0 \mathrm{~nm}$ (no particle) to $12 \mathrm{~nm}$ with increment of $0.5 \mathrm{~nm}$ for $\mathrm{p}$ - and s-polarization, respectively. exponentially due to the absorption in mental bulk and the destructive interference of SPPs between particle to particle interactions, while it may increase by plasmon resonance by controlling the diameter size in the range of $1-7 \mathrm{~nm}$. The field intensity at different particle diameters exhibited double peaks which were the points of surface plasmon resonance occurred, one was around $3 \mathrm{~nm}$ and the other was $6 \mathrm{~nm}$. This indicates that the particle diameter of $3 \mathrm{~nm}$ and $6 \mathrm{~nm}$ provide a major contribution to plasmon coupling with the dipole radiation between particle to particle is the evanescent field of near-field photons. Since the field propagation is dominantly taken with the near-field photons, super-resolution exceeding the diffraction limit is made similar to that of near-field scanning optical microscopy.

As excepted, the field intensity shown in Fig. 4b for spolarization decays gradually as the diameters of $\mathrm{Ag}$ nano-particles increases and can not excite SPPs when the diameter of $\mathrm{Ag}$ nano-particles less than $4 \mathrm{~nm}$. It should be noted that there is a further point which needs to be clarified, i.e., the field intensity for s-polarization larger than the p-polarization when the diameter of Ag nano-particles larger than $4 \mathrm{~nm}$. In Fig. $4 \mathrm{~b}$, the field intensity for s-polarization increases and reaches maximum value at $5 \mathrm{~nm}$ and then decays slowly as the diameter increases. The phenomenon then arises about the $\mathrm{AgOx}$ layer can act as a bulk material when the diameters of Ag nano-particles larger than $4 \mathrm{~nm}$ which embedded in the finite region with finite depth of $15 \mathrm{~nm}$ in active layer (AgOx). To explain this phenomenon, we can consider that the boundary condition and the incident wave should have a component vertical to the active layer in order to excite the localized-mode surface plasmon [19].

Fig. 5a shows an example of Fig. 4 that the field intensity at the wavelength of incident light of $\lambda=405 \mathrm{~nm}$. Fig. $5 \mathrm{~b}$ and $\mathrm{c}$ show the calculated results of fragment region of $3 \mathrm{D}$ near-field distribution (contour map of $E_{y}$ in $x-z$ plane at $y=0$ ) for (a) s-polarization and (b) p-polarization illuminations, respectively. We choose the diameter of $\mathrm{Ag}$ nano-particles is $7 \mathrm{~nm}$ and wavelength of $\lambda=405 \mathrm{~nm}$ as an example. It is clearly seen from Fig. $5 b$ for s-polarization that no SPPs was excited. Compared to the results shown in Fig. 5c for p-polarization, more SPPS was excited. In the central part of active layer shown in Fig. $5 b$, the electric intensity is stronger than those in other region of the layer. Besides, It is found that the decrease in the images contrast relative to the image shown in Fig. 5c. In this case, the s-polarization is responsible for the surface digging. The estimated digging of the surface after illumination by a metallic bulk formed by larger diameter of $\mathrm{Ag}$ nano-particles gathered in $\mathrm{AgOx}$ layer with s-polarization is similar to the experimental surface deformation in good agreement with the observation in our current experimental data. The main differences between the two principal polarizations are hence the degree of confinement which is favorable for s-polarization and the available electric field distribution and SPPs which is favorable for 


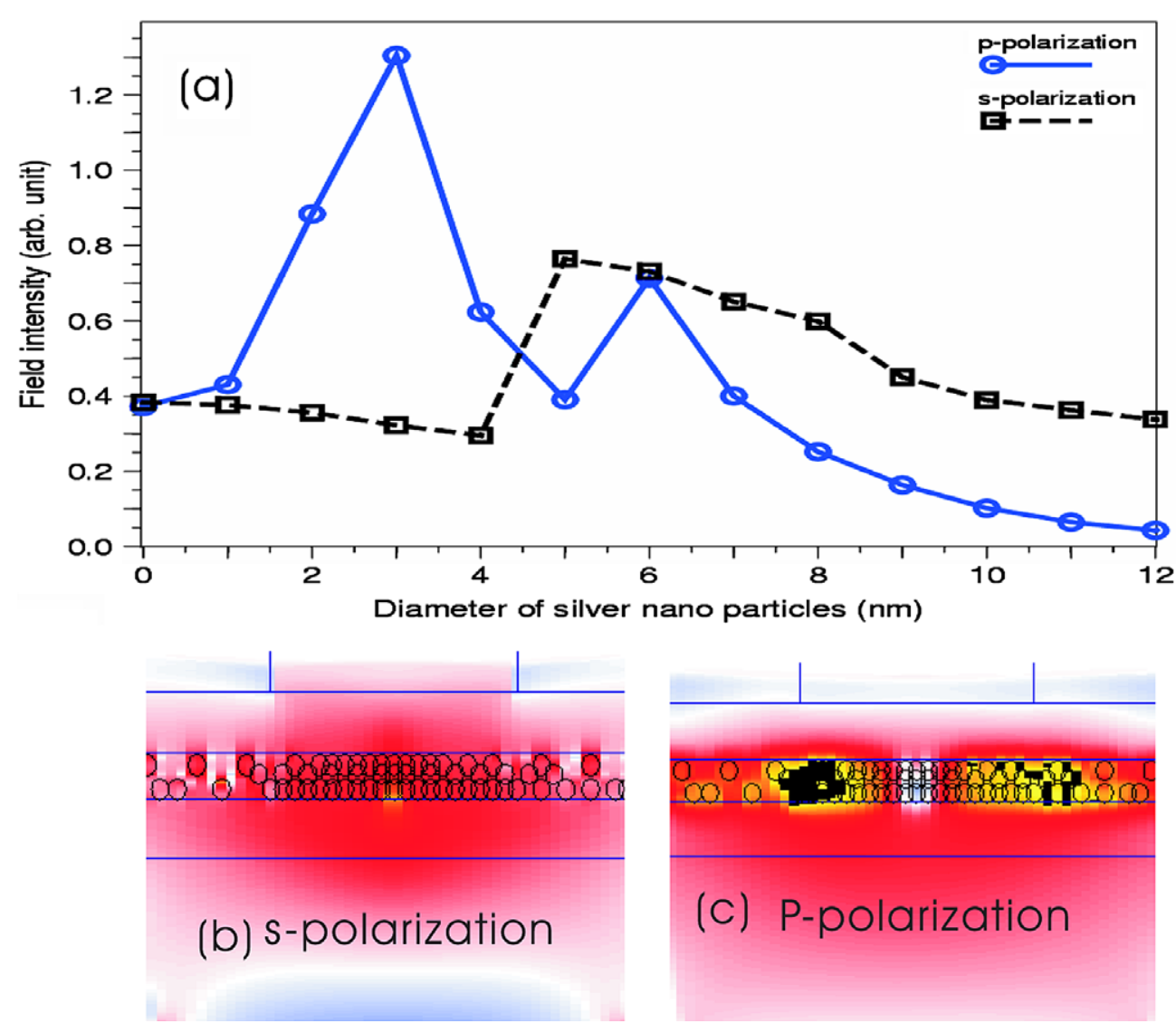

Fig. 5. (a) An example of Fig. 4 that the field intensity at the wavelength of incident light of $405 \mathrm{~nm}$; (b) and (c) are calculated results of the fragment region of the 3D near-field distribution (contour map of $E_{y}$ in $x-z$ plane at $y=0$ ) for (b) s-polarization and (c) p-polarization illuminations, respectively. The diameter of $\mathrm{Ag}$ nano-particles is $7 \mathrm{~nm}$ and the wavelength of incident wave is $\lambda=450 \mathrm{~nm}$ as an example.

p-polarization. It is important to remark here that the intensity of p-polarized field plays a leading role. If we come back to the field maps of Fig. $5 \mathrm{~b}$ and $\mathrm{c}$, we see that the SPPs is generated mostly by the surface of Ag nanoparticles in p-polarization, coming from the excitation of the metal doping and the apparition of a local dipole along the $\mathrm{AgOx}$ layer. On the contrary, for s-polarization, there is a lot of light at the center part of $\mathrm{AgOx}$ layer, is responsible for the surface digging. And the results is good agreement to our previous study as described in Ref. [20].

It is necessary to note that the phase-change material GST is utilized as the recording medium due to its fast and stable performance and large reflective changes between the crystalline and amorphous states [21,22]. Recently, a local change in optical constant caused by laser heating of the phase-change layer has reported to play an important role in readout $[23,24]$. Thus, temperature effects inside the disc must be considered to understand the readout phenomena [25]. The active layer of the Super-RENS structure is deformed (bubble pit shape) after a writing process, as observed in experimental work $[26,27]$ and theoretical analysis [28]. In the further research, we will think about the case where the temperature distribution inside the disc. Further research on the effects of different distribution patterns may yield a better understanding of the optical properties of the deformed AgOx-type or PtOx type Super-RENS. Moreover, on the basis of results of this study, we will calculate the change in intensity (difference signal) in the far-field that becomes detectable signals.

In conclusion, the nonlinear near-field optical properties of AgOx super-RENS structure have been studied by use of 3D FDTD simulations. We find that the polarization direction, wavelength of incident light, and the size of Ag nanoparticles are sensitive to the plasma resonance. The 3D model can effectively predict the nonlinear behavior of the AgOx super-RENS structure and obtain more information than the $2 \mathrm{D}$ one due to the resolution provided by the 2D model Super-RENS is lower than that expected on the basis of experiments. With above research can help us to design a super-RENS with superior resolution as well as an alternative approach to near-field optical research, plasmon device, biological sensors and nano-photonic devices.

\section{Acknowledgements}

The authors are thankful for the financial support from National Science Council, Taiwan, ROC, under Grant number NSC 94-2112-M-231-001, and the Ministry of Economic Affairs, Taiwan, ROC, under Grant number 94-EC17-A-08-S1-0006. 


\section{References}

[1] W.C. Liu, C.Y. Wen, K.H. Chen, W.C. Lin, D.P. Tsai, Appl. Phys. Lett. 78 (2001) 685.

[2] H. Fuji, J. Tominaga, L. Men, T. Nakano, H. Katayama, N. Atado, Jpn. J. Appl. Phys. 39 (2000) 980.

[3] W.C. Hsu, M.R. Tseng, S.Y. Tsai, P.C. Kuo, Jpn. J. Appl. Phys. 42 (2003) 1005.

[4] H. Kim, I. Hwang, J. Kim, C. Park, M. Ro, J. Lee, M. Jung, I. Park, Jpn. J. Appl. Phys. 44 (2005) 3605.

[5] H. Kim, J. Kim, C. Park, M. Jung, M. Ro, I. Park, Jpn. J. Appl. Phys. 45 (2006) 1374

[6] S.Y. Kim, S.U. Park, X.Z. Li, S.J. Kim, S.H. An, Jpn. J. Appl. Phys. 45 (2006) 1390

[7] J. Kim, H. Fuji, Y. Yamakawa, T. Nakano, D. Buechel, J. Tominaga, N. Atoda, Jpn. J. Appl. Phys. 40 (2001) 1634.

[8] J. Tominaga, T. Nakano, N. Atoda, Appl. Phys. Lett. 73 (1998) 2078.

[9] T.C. Chu, W.C. Liu, D.P. Tsai, Opt. Comm. 246 (2005) 561.

[10] W.C. Liu, M.Y. Ng, D.P. Tsai, Jpn. J. Appl. Phys. 43 (2004) 4713.

[11] W.C. Liu, D.P. Tsai, Jpn. J. Appl. Phys. 42 (2003) 1031.

[12] D.A. Schultz, Curr. Opin. Biotechnol. 14 (2003) 13

[13] M. Ohtsu, K. Kobayashi, T. Kawazoe, S. Sangu, T. Yatsui, IEEE J. Sel. Top. Quantum Electron. 8 (2002) 839.

[14] S.A. Maier, M.L. Brongersma, P.G. Kik, S. Meltzer, A.A.G. Requicha, H.A. Atwater, Adv. Mater 13 (2001) 1501.
[15] J.B. Judkins, R.W. Ziolkowski, J. Opt. Soc. Am. A 12 (1995) 1974.

[16] S.G. Stan, The CD-ROM Drive; A Brief System Description, Kluwer Academic Publishers., Boston, 1998 (Chapter 2.3, p. 16).

[17] T. Nakano, A. Sato, H. Fuji, J. Tominaga, N. Atoda, Appl. Phys Lett. 75 (1999) 151.

[18] M.Y. Ng, W.C. Liu, Opt. Express 14 (2005) 9422.

[19] H. Raether, Surface Plasmons on Smooth and Rough Surfaces and on Gratings, Springer, Berlin, 1988.

[20] Y.F. Chau, T.J. Yang, D.P. Tsai, Jpn. J. Appl. Phys. 43 (2004) 8115.

[21] A.V. Kolbov, P. Fons, J. Tominaga, A.I. Frenkel, A.L. Ankudinov, S.N. Yannopoulos, K.S. Andrikopoulos, T. Uruga, Jpn. J. Appl. Phys. 44 (2005) 3345

[22] A.V. Kolobov, P. Fons, J. Tominaga, A.L. Ankudinov, S.N. Yannopoulos, K.S. Andrikopoulos, J. Phys. Condens. Matter 16 (2004) S5103.

[23] J. Tominaga, T. Shima, M. Kuwahara, T. Fukaya, A.V. Kolobov, T. Nakano, Nanotechnology 15 (2004) 411.

[24] M. Kuwahara, T. Shima, A. Kolobov, J. Tominaga, Jpn. J. Appl. Phys. 43 (2004) L8.

[25] Y. Yamakawa, K. Kurihara, M. Kuwahara, T. Shima, T. Nakano, J. Tominaga, Jpn. J. Appl. Phys. 45 (2006) 1463.

[26] T. Kikukawa, A. Tachibana, H. Fuji, J. Tominaga, Jpn. J. Appl. Phys. 42 (2003) 1038.

[27] H. Fuji, T. Kikukawa, J. Tominaga, Jpn. J. Appl. Phys. 43 (2004) 4212.

[28] K. Kataja, J. Olkkonen, ISPS, 2005, 9. 